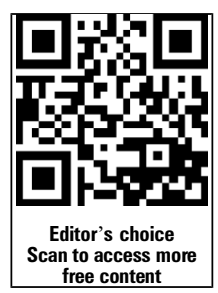

${ }^{1}$ Division of Occupational and Environmental Medicine, UConn Health Center, Farmington, Connecticut, USA ${ }^{2}$ Department of Environmental Health, Harvard T.H. Chan School of Public Health, Boston, Massachusetts, USA ${ }^{3}$ School of Public Health, University of California, Berkeley, California, USA ${ }^{4}$ Cardiovascular Epidemiology Research Unit, Beth Israel Deaconess Medical Center and Harvard Medical School, Boston, Massachusetts, USA ${ }^{5}$ Department of Epidemiology, Harvard T.H. Chan School of Public Health, Boston, Massachusetts, USA ${ }^{6}$ Massachusetts General Hospital, Boston, Massachusetts, USA

\section{Correspondence to} Dr Jennifer M Cavallari, Division of Occupational and Environmental Medicine, UConn Health Center, Farmington 06032, CT, USA; cavallari@uchc.edu

Received 11 August 2015 Revised 7 March 2016 Accepted 18 March 2016 Published Online First 6 April 2016

\title{
Environmental and occupational particulate matter exposures and ectopic heart beats in welders
}

\author{
Jennifer M Cavallari, ${ }^{1,2}$ Shona C Fang, ${ }^{2}$ Ellen A Eisen, ${ }^{3}$ Murray A Mittleman, ${ }^{4,5}$ \\ David C Christiani ${ }^{2}, 5,6$
}

\section{ABSTRACT \\ Objectives Links between arrhythmias and particulate matter exposures have been found among sensitive populations. We examined the relationship between personal particulate matter $\leq 2.5 \mu \mathrm{m}$ aerodynamic diameter $\left(\mathrm{PM}_{2.5}\right)$ exposures and ectopy in a panel study of healthy welders.}

Methods Simultaneous ambulatory ECG and personal $\mathrm{PM}_{2.5}$ exposure monitoring with DustTrak Aerosol Monitor was performed on 72 males during work and non-work periods for 5-90 h (median $40 \mathrm{~h}$ ). ECGs were summarised hourly for supraventricular ectopy (SVE) and ventricular ectopy (VE). PM $_{2.5}$ exposures both work and non-work periods were averaged hourly with lags from 0 to $7 \mathrm{~h}$. Generalised linear mixed-effects models with a random participant intercept were used to examine the relationship between $\mathrm{PM}_{2.5}$ exposure and the odds of SVE or VE. Sensitivity analyses were performed to assess whether relationships differed by work period and among current smokers.

Results Participants had a mean (SD) age of 38 (11) years and were monitored over 2993 person-hours. The number of hourly ectopic events was highly skewed with mean (SD) of 14 (69) VE and 1 (4) SVE. We found marginally significant increases in VE with $\mathrm{PM}_{2.5}$ exposures in the sixth and seventh hour lags, yet no association with SVE. For every $100 \mu \mathrm{g} / \mathrm{m}^{3}$ increase in sixth hour lagged $\mathrm{PM}_{2.5}$, the adjusted OR $(95 \% \mathrm{Cl})$ for VE was 1.03 (1.00 to 1.05). Results persisted in work or non-work exposure periods and non-smokers had increased odds of VE associated with $\mathrm{PM}_{2.5}$ as compared with smokers.

Conclusions A small increase in the odds of VE with short-term $\mathrm{PM}_{2.5}$ exposure was observed among relatively healthy men with environmental and occupational exposures.

\section{INTRODUCTION}

The American Heart Association has deemed particulate matter $\leq 2.5 \mu \mathrm{m}$ aerodynamic diameter $\left(\mathrm{PM}_{2.5}\right)$ exposure as a modifiable risk factor contributing to cardiovascular morbidity and mortality. ${ }^{1}$ While the exact mechanisms responsible for this association remain under investigation, research suggests that sympathetic activation and parasympathetic suppression is one pathway by which particulate matter (PM) can elicit a cardiovascular response. ${ }^{2}$ It is plausible that the particle-induced autonomic nervous system imbalance may result in a decreased threshold for arrhythmias. ${ }^{3}$ A number of epidemiological studies have focused on

\section{What this paper adds}

- Particulate matter (PM) exposure has been linked with arrhythmia, primarily in sensitive populations exposed to ambient PM.

- In a cohort of healthy, male boilermaker welders exposed to both ambient and occupational sources of PM, this study investigates the association between ventricular and supraventricular ectopic beats and PM exposures.

- A modest increase in risk of ventricular ectopy was associated with PM exposure in the preceding 6-7 h. While associations were similar regardless of exposure period (work or non-work), smoking status appeared to impact risk, with non-smokers being more susceptible.

investigating the ability of air pollution to trigger cardiac arrhythmias. ${ }^{4-6}$

Among individuals with implanted cardioverter defibrillators (ICDs), epidemiological investigations have revealed associations between ambient particle exposures and ICD-recorded arrhythmia events, commonly tachyarrhythmias, in many populations. $^{7-10}$ Not all studies of ICD patients have observed associations with $\mathrm{PM},{ }^{11-14}$ possibly due to differences in particle source, concentration levels, as well as differences in study designs and methods. ${ }^{1}$

The association between PM exposures and arrhythmia, specifically ectopy, in healthy individuals has been investigated in a small number of studies. Associations between ectopy and PM exposure have been observed in a panel study of healthy patrol troopers, ${ }^{15}$ postmenopausal women within the Women's Health Initiative Study, ${ }^{16}$ elderly patients within the Normative Aging Study, ${ }^{6}$ and middle-aged, healthy non-smokers within the Air Pollution on Cardiac Arrhythmia (APACR) study. ${ }^{5}$ The association between ectopy and PM exposure in populations with both occupational and environmental exposures has yet to be investigated.

This study was designed to investigate whether $\mathrm{PM}_{2.5}$ exposures are associated with increases in arrhythmia events, specifically ectopy, among a relatively healthy population of boilermaker construction workers, or 'boilermakers', exposed to
Fang SC, Eisen EA, et al.

Occup Environ Med 
PM from both occupational and non-occupational (environmental) sources. Boilermakers are construction workers who are exposed to metal-rich welding fumes when they build, maintain and repair industrial boilers that are often located in power plants. In our previous work within a boilermaker population, we observed associations between cardiac autonomic control, measured using heart rate variability, and $\mathrm{PM}_{2.5}$ exposures. ${ }^{17-21}$ However, the associations between $\mathrm{PM}_{2.5}$ exposures and arrhythmia events have not been examined within this cohort. Using a repeated measures panel study design where each worker serves as his own control, and with simultaneous ECG and $\mathrm{PM}_{2.5}$ exposure monitoring, we examined whether $\mathrm{PM}_{2.5}$ exposures were associated with increased odds of ectopy and whether different exposure-related factors modified this association.

\section{METHODS}

\section{Study design and population}

From 1999 to 2006, we recruited 72 male boilermakers from a local union into a panel study and monitored participants by ambulatory ECG to assess cardiac responses to PM exposure. Sampling campaigns were performed approximately yearly, and some workers $(35 \%)$ participated in the study on more than 1 year. Each year, participants were monitored over work and non-work days from 5 to $90 \mathrm{~h}$ (median of $42 \mathrm{~h}$ yearly). Workday monitoring occurred while participants trained at an apprentice welding school or while working on the repair and maintenance of an oil-fired boiler. Apprentice school monitoring occurred in 1999, 2003, 2004, 2005 and 2006 in the winter and summer months, when the majority of workers were not actively working outside the union hall. The welding school consisted of one large, temperature-controlled room with 10 workstations with local exhaust ventilation where the boilermakers received instruction and practiced welding, cutting and grinding techniques. Monitoring at the oil-fired boiler overhaul site occurred in the Spring of 1999 and Fall of 2000. The boiler overhaul entailed removing and replacing the boiler's interior wall panels and water-circulating tubing. At both locations, boilermakers primarily performed shielded metal arc welding (stick) and gas metal arc welding, most commonly using base metals of mild steel (manganese alloys) and stainless steel (manganese, chromium and nickel alloys) with electrodes composed mainly of iron with variable amounts of manganese (1-5\%). Plasma arc or acetylene torch cutting and grinding also occurred at both work sites. In addition to workday monitoring, workers were also monitored while they were not working or training as they went about their normal activities on a non-workday.

A self-administered modified American Thoracic Society questionnaire $^{22}$ was used to collect information on medical history and medication use. Participants were classified as having a cardiac condition if they reported any of the following: heart trouble, congestive heart failure, angina, arrhythmia, myocardial infarction, heart/chest operation or otherwise non-classified heart problems. Hypertension was classified as a reported doctor diagnosis or the use of hypertensive medications including ACE inhibitors or $\beta$-blockers. Demographic and lifestyle information including smoking history and occupational history was also collected.

\section{Continuous ambulatory ECG and arrhythmias}

Prior to the start of work, participants were fitted with a standard five-lead ECG Holter monitor (Cardio Data Systems, Dynacord model 423). To facilitate good lead contacts, the participant's skin was shaved, if necessary, cleansed with an alcohol wipe, and slightly abraded. Study staff checked leads at the workplace periodically. Each tape was sent to Raytel Cardiac Services (Haddonfield, New Jersey, USA) for processing and analysis using a DelMar Avionic (Irvine, California, USA) Model Strata Scan 563. Trained technicians, blinded to the exposure levels, used standard criteria to accept or reject all normal or abnormal findings. Ventricular and supraventricular arrhythmia events (yes/no) were summarised hourly. In addition, the total number of supraventricular events (SVEs) and ventricular events (VEs), quantity of couplets and triplets, and the presence of ventricular and supraventricular tachyarrhythmias were reported. High VE responders were identified as those individuals whose median hourly quantity of VE beats over the study period was greater than the 95 th centile for the population ( 20 beats/h).

\section{PM exposures}

Personal $\mathrm{PM}_{2.5}$ exposures were continuously monitored using the light-scattering technology of a DustTrak Aerosol Monitor (TSI, Inc, St Paul, Minnesota, USA) fitted with a $\mathrm{PM}_{2.5}$ inlet impactor. The DustTrak was placed in a padded pack, with the inlet tubing secured to the participant's shoulder in the breathing zone area. The participants wore the pack throughout the work day. At the end of the work shift, participants were asked whether they used a respirator and the work shift start and end times were recorded.

Participants were asked to wear the pack during non-workday monitoring as well. Participants were instructed to keep the inlet tube near their breathing zone during everyday activities and to place the pack on the bedside while sleeping.

The DustTrak received yearly factory calibration and daily zero balance and flow checks. A previous study of welding fume exposures validated the use of the DustTrak to capture welding fume exposures. ${ }^{23}$ The DustTrak took $\mathrm{PM}_{2.5}$ concentration readings every $10 \mathrm{~s}$ and recorded $1 \mathrm{~min}$ averages. One-hour averages were calculated from the 1 min $\mathrm{PM}_{2.5}$ concentrations.

\section{Statistical analysis}

To understand the relationship between ectopy and $\mathrm{PM}_{2.5}$ exposure, the hourly outcome data were modelled using generalised linear mixed-effects models in two ways: as a binary outcome indicating the presence of one or more ectopic beat within the hour and as count data in beats per hour.

In the first analysis, the odds of having a SVE or VE (at least one ectopic beat within a $1 \mathrm{~h}$ period) for every unit increase in $\mathrm{PM}_{2.5}$ (environmental and occupational) was investigated using a logistic regression model with a random intercept for each participant to account for repeated measurements. Potential confounders were evaluated by considering adjustment for predictors of the outcome including age, current smoker (yes/no), time of day; and correlates of exposure including season (winter vs summer), worksite location (power plant vs welding school), and time-varying indicators for whether the participant was at work and/or wore a respirator over the work shift. Only statistically significant predictors were retained in the final model (age, season and worksite location). We evaluated the use of splines for age and time of day, but found that the linear trend for age and three categories for time of day (8:00-15:59; $16: 00-23: 59 ; 0: 00-7: 59)$ best fit the data.

We considered hourly $\mathrm{PM}_{2.5}$ concentrations within the same hour as the arrhythmia outcomes as well as with lags up to $7 \mathrm{~h}$ preceding the arrhythmia event. Since $\mathrm{PM}_{2.5}$ and arrhythmia events were monitored simultaneously, the introduction of exposure lags resulted in missing data. A priori, we wanted to fit models using a minimum of $75 \%$ of the data; therefore, lags 
longer than $7 \mathrm{~h}$ were not considered. The lag structure for the exposure was evaluated separately and jointly in a polynomial distributed lag model. ${ }^{24}$

We explored the robustness of our finding in a series of sensitivity analyses. First, we evaluated the effect of including all potential confounders regardless of statistical significance. We also investigated whether controlling for heart rate, a potential surrogate of activity level and stress, affected study results. We explored whether individuals experiencing a high number of ectopic beats as well as participants reporting a cardiac condition were driving study results by excluding these individuals from the analysis. We examined the change in effect estimate after excluding participants who experienced (1) ectopic beats during each hourly monitoring period and (2) no ectopic beats during the monitoring period as they would have no variation in their outcome. Lastly, we examined whether boilermakers who participated on multiple occasions were influencing the results by only including data from first participation.

For the second analysis, given the overdispersion of the ectopic beat data, generalised linear mixed-effects models with random intercepts for each individual, a log-link function and a negative binomial distribution assumption were used to examine the relationship between the number of beats per hour and hourly $\mathrm{PM}_{2.5}$ exposure.

To explore exposure-related factors effecting the association between $\mathrm{PM}_{2.5}$ and $\mathrm{VE}$, we incorporated an interaction term between the $\mathrm{PM}_{2.5}$ variable and different exposure characteristics including whether the participant was at work, wore a respirator, worked the prior day or was a smoker. The detail and time scale for each of these exposure-related variable was limited. Work was a dichotomous variable that was yes when the $\mathrm{PM}_{2.5}$ exposure was collected during work hours. Similarly,

Table 1 Clinical and demographic characteristics of the 72 boilermaker construction worker participants

\begin{tabular}{|c|c|c|}
\hline Observation hours & & 2993 \\
\hline \multicolumn{3}{|l|}{ Arrhythmia events } \\
\hline \multicolumn{3}{|l|}{ Ventricular (beats/h) } \\
\hline & Mean (SD) & $14(69)$ \\
\hline & Minimum, median, maximum & $0,0,877$ \\
\hline \multicolumn{3}{|l|}{ Supraventricular (beats/h) } \\
\hline & Mean (SD) & $1(4)$ \\
\hline & Minimum, median, maximum & $0,0,92$ \\
\hline \multicolumn{3}{|l|}{ General* $^{*}$} \\
\hline Male & $\mathrm{n}(\%)$ & $72(100)$ \\
\hline \multirow[t]{2}{*}{ Age (years) } & Mean (SD) & $38(11)$ \\
\hline & Range & $19-60$ \\
\hline Current smoker n (\%) & & $31(43)$ \\
\hline \multirow[t]{2}{*}{ Years as a boilermaker } & Mean (SD) & $10(12)$ \\
\hline & Range & $0-40$ \\
\hline Diagnosest & $\mathrm{n}(\%)$ & \\
\hline \multicolumn{3}{|l|}{ Cardiovascular } \\
\hline Angina pectoris & & $1(1)$ \\
\hline Ischaemic heart disease & & $3(4)$ \\
\hline Myocardial infarction & & $4(6)$ \\
\hline Hypertension & & $17(24)$ \\
\hline \multicolumn{3}{|l|}{ Respiratory } \\
\hline Chronic bronchitis & & $9(13)$ \\
\hline Asthma & & $5(7)$ \\
\hline
\end{tabular}

respirator use was a dichotomous variable that was yes during work hours if the participant reported wearing a respirator. If the participant worked the prior day, the dichotomous variable was coded yes. Current smokers were coded yes.

We present ORs and rate ratio (RR) and 95\% CIs based on a $100 \mu \mathrm{g} / \mathrm{m}^{3}$ increase in $\mathrm{PM}_{2.5}$ exposure, and set $\mathrm{p}$ values $<0.05$ as statistically significant. Analyses were performed using the NLMIXED procedure in SAS (SAS Institute, Cary, North Carolina, USA).

\section{RESULTS}

From 1999 to 2006, we collected ECG data on 72 participants. Participants were monitored from 1-24 (median 15) hours daily. Workday monitoring occurred on all participants and $51(71 \%)$ were also monitored on a non-workday. Workers were allowed to participate on multiple occasions over the 7-year study duration, although the majority $65 \%(n=47)$ participated once with $25 \%(n=18)$ twice and $10 \%(n=7)$ on three or more occasions. The full data set contained $2993 \mathrm{~h}$ of simultaneous arrhythmia and $\mathrm{PM}_{2.5}$ exposure monitoring. When exposure was lagged by $6 \mathrm{~h}$, there were $2356 \mathrm{~h}$ from each of the 72 participants.

Outcome and population characteristics are summarised in table 1. The overwhelming majority of arrhythmia events experienced by the population were single ectopic beats, although there were 13 incidents of tachycardia, 14 couplet and 1 triplet events which were excluded from the analysis due to small numbers. Fifty-eight participants experienced VEs and 68 SVEs over the study period. In total, the population experienced VE and SVE during 671 and 784 h, respectively, while monitored, with a large variability in the quantity of hourly beats with a mean (SD) of 14 (69) and 1 (4) for ventricular and supraventricular, respectively. Twelve participants had at least $1 \mathrm{~h}$ with 12 or more VE and were classified as high VE responders in the sensitivity analysis.

The participants were all male with a wide range of ages from 19 to 60 years, mean (SD) of 38 (11) years. On first enrolment, $43 \%$ were current smokers and among the non-smokers, 4 $(10 \%)$ had previously smoked. Some participants reported cardiovascular diseases with $24 \%$ reporting hypertension. Twelve (17\%) participants reported having one or more cardiac conditions.

Personal $\mathrm{PM}_{2.5}$ exposures were log normally distributed with a mean (SD) of $274(754) \mu \mathrm{g} / \mathrm{m}^{3}$ and median of $41 \mu \mathrm{g} / \mathrm{m}^{3}$ over the entire period (table 2). Exposure levels were nearly 20 times higher when working (median of $420 \mu \mathrm{g} / \mathrm{m}^{3}$ ) as compared with when participants were not working (median of $26 \mu \mathrm{g} / \mathrm{m}^{3}$ ) and were higher among smokers as compared with non-smokers. The non-working, personal $\mathrm{PM}_{2.5}$ exposures experienced by this population are similar to the personal $\mathrm{PM}_{2.5}$ levels reported for 212 non-smoking individuals living across four areas of the USA (median of $31 \mu \mathrm{g} / \mathrm{m}^{3}$ ). ${ }^{25}$

The individual, lagged effects of $\mathrm{PM}_{2.5}$ on having one or more VE or SVE are presented in figure 1. In summary, we found a statistically significant $(\mathrm{p}<0.05)$ exposure-related increased risk of having a VE 6 and $7 \mathrm{~h}$ following exposure. We observed no association between SVE and hourly $\mathrm{PM}_{2.5}$ exposure up to $7 \mathrm{~h}$ following exposure. We observed no statistically significant association or trend when we examined the cumulative effect of exposure, incorporating each hourly $\mathrm{PM}_{2.5}$ from concurrent to $7 \mathrm{~h}$ prior to each VE in cumulative distributed lag models (data not shown).

In sensitivity analyses, we examined the association between $6 \mathrm{~h}$ lagged $\mathrm{PM}_{2.5}$ and $\mathrm{VE}$ (table 3). This association did not 
Table 2 Personal particulate matter $\leq 2.5 \mu \mathrm{m}$ aerodynamic diameter concentrations $\left(\mu \mathrm{g} / \mathrm{m}^{3}\right)$ from DustTrak aerosol monitor over the monitoring period

\begin{tabular}{|c|c|c|c|c|c|c|c|c|}
\hline & \multicolumn{4}{|c|}{ Lag $0^{*}$} & \multicolumn{4}{|c|}{ Lag $6 \dagger$} \\
\hline & $\mathrm{N}$ & Median & Mean (SD) & Q25, Q75 & $\mathbf{N}$ & Median & Mean (SD) & Q25, Q75 \\
\hline Entire period & 2993 & 41 & $274(754)$ & 13,186 & 2356 & 48 & $300(48)$ & 14,229 \\
\hline Not working & 2331 & 26 & 123 (496) & 10,70 & 1765 & 28 & 154 (581) & 10,84 \\
\hline Working & 662 & 420 & $804(1162)$ & 146,1104 & 591 & 404 & 736 (909) & 143,1060 \\
\hline Smokers & 1124 & 79 & 422 (1077) & 26,354 & 866 & 111 & $456(1004)$ & 26,354 \\
\hline Non-smokers & 1869 & 28 & $185(439)$ & 10,103 & 1490 & 32 & $209(471)$ & 10,128 \\
\hline
\end{tabular}

*Lag 0 represents concurrent exposure and arrhythmia monitoring.

tLag 6 is the exposure in the $6 \mathrm{~h}$ prior to ectopy monitoring.

change when the analysis was performed with additional adjustment for potential confounders or for heart rate. Likewise, the association persisted when the high VE responders, those presenting with a cardiac condition, or participants with constant $(n=3)$ or no ectopy response $(n=14)$ were removed from the analysis. When we restricted the analysis to the first participation occasion, we observed little change in the effect estimate, although the CIs widened due to small sample size.

We observed no association between the quantity of hourly $\mathrm{VE}$ and $\mathrm{PM}_{2.5}$ exposure; adjusted RR of 0.989 (95\% CI 0.974 to 1.003$) \mathrm{VE}$ per hour per $100 \mu \mathrm{g} / \mathrm{m}^{3}$ increase in $\mathrm{PM}_{2.5} 6 \mathrm{~h}$ prior.

Using an interaction term, we examined whether the odds of VE differed by exposure and lifestyle characteristics (figure 2). When we distinguished between periods of work versus non-work periods, the association persisted. In fact, for a $100 \mu \mathrm{g} / \mathrm{m}^{3}$ increase in $\mathrm{PM}_{2.5}$ in the prior $6 \mathrm{~h}$, we observed the same increased odds of VE during non-work (OR 1.03, 95\% CI 0.99 to 1.06 ) as compared with during work (OR 1.03, 95\% CI 0.99 to 1.06 ). The difference between these effect estimates was not statistically significant, nor was there a difference in baseline odds of VE under the two conditions. Respirator use did not predict VE, nor was there a statistically significant difference between those who reported using a respirator (OR 1.02, 95\% CI 0.95 to 1.10 ) and those who did not (OR 1.02, 95\% CI 1.00 to 1.05$)$. Likewise, we observed no difference among participants who reported working on the day prior to monitoring (OR 1.03, 95\% CI 0.99 to 1.07 ) as compared with those who

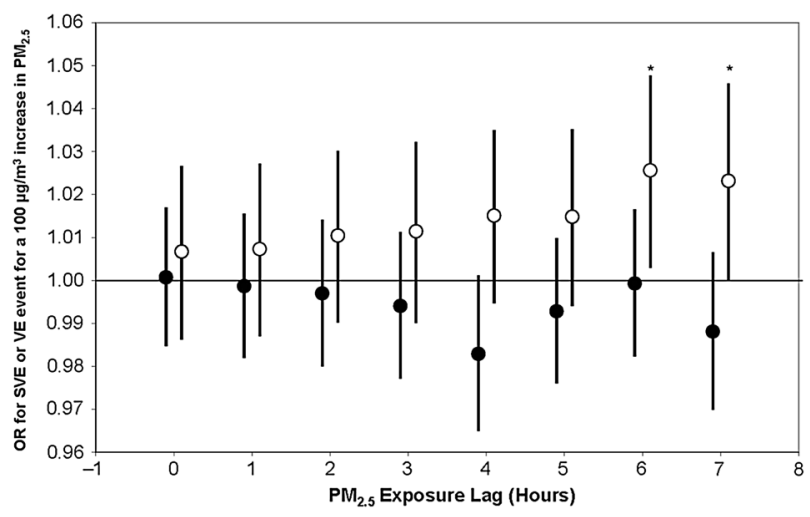

Figure 1 ORs for supraventricular event (solid circle) and ventricular event (open circle) hourly per $100 \mu \mathrm{g} / \mathrm{m}^{3}$ increase in $\mathrm{PM}_{2.5}$ exposure lagged from 0 to $7 \mathrm{~h}$. All models are adjusted for age, time of day, season and worksite. Error bars indicate $95 \% \mathrm{Cls}$. $\mathrm{p}<0.05$. $\mathrm{PM}_{2.5}$ particulate matter $\leq 2.5 \mu \mathrm{m}$ aerodynamic diameter; SVE, supraventricular ectopy; VE, ventricular ectopy. were not working the prior day (OR 1.03, 95\% CI 1.00 to 1.05). We observed a statistically significant difference in the exposure-response relationship between smokers and nonsmokers with an increased odds with non-smokers (OR 1.07, $95 \%$ CI 1.03 to 1.11 ) as compared with smokers (OR 1.00, 95\% CI 0.97 to 1.03 ) (figure 2). Furthermore, non-smokers had an increased baseline risk of experiencing a VE over the study period after adjusting for covariates including $\mathrm{PM}_{2.5}$ (OR 1.10, $95 \%$ CI 0.98 to 1.23$)$, although the risk was not statistically significant $(\mathrm{p}=0.10)$.

\section{DISCUSSION}

In a population of healthy male workers, we observed a small but statistically significant $(3 \%)$ increased odds of experiencing a VE for every $100 \mu \mathrm{g} / \mathrm{m}^{3}$ increase in $\mathrm{PM}_{2.5}$ exposure in the prior $6 \mathrm{~h}$, yet no association with SVE. This association was stable to adjustment by a number of potential confounders.

Our findings are consistent with other studies of healthy populations that observed particle-related arrhythmia response of ectopy. ${ }^{51526}$ Persistent ventricular ectopy is associated with increased risk of all-cause and cardiovascular mortality ${ }^{27}$; however, it is a common event among the general population, and the clinical significance of the particle-related increased ventricular ectopy in healthy individuals remains unclear. More clinically relevant events have been observed in compromised populations, where associations between PM exposures and activity of implantable cardioverter defibrillators (ICDs)devices used in persons who have experienced or are at risk for life-threatening ventricular arrhythmias-have been

Table 3 Sensitivity analyses presenting adjusted OR per $100 \mu \mathrm{g} / \mathrm{m}^{3}$ increase in $\mathrm{PM}_{2.5}$ in $6 \mathrm{~h}$ prior

\begin{tabular}{llll}
\hline & $\mathbf{n}$ & $\begin{array}{l}\text { Adjusted } \\
\text { OR* }\end{array}$ & $\mathbf{9 5 \% ~ C l}$ \\
\hline $\begin{array}{l}\text { Original model } \\
\text { Additional covariates }\end{array}$ & 2356 & 1.03 & 1.00 to 1.05 \\
$\quad$ Working, respirator use & 2356 & 1.03 & 1.00 to 1.05 \\
$\quad$ Heart rate & 2356 & 1.03 & 1.00 to 1.05 \\
$\quad$ Restrictions & & & \\
$\quad$ Excluding high VE responders & 2199 & 1.03 & 1.00 to 1.05 \\
$\quad$ Excluding cardiac compromised & 1950 & 1.02 & 1.00 to 1.04 \\
$\quad$ Excluding constant and non-responders & 2074 & 1.02 & 1.00 to 1.04 \\
$\quad$ First participation time only & 734 & 1.02 & 0.99 to 1.05
\end{tabular}

* Estimates the odds of having one or more VE in an hour period for $100 \mu \mathrm{g} / \mathrm{m}^{3}$ increase in $\mathrm{PM}_{2.5}$ after adjusting for age, time of day, season and worksite. $\mathrm{PM}_{2.5}$, particulate matter $\leq 2.5 \mu \mathrm{m}$ aerodynamic diameter; $\mathrm{VE}$, ventricular event. 


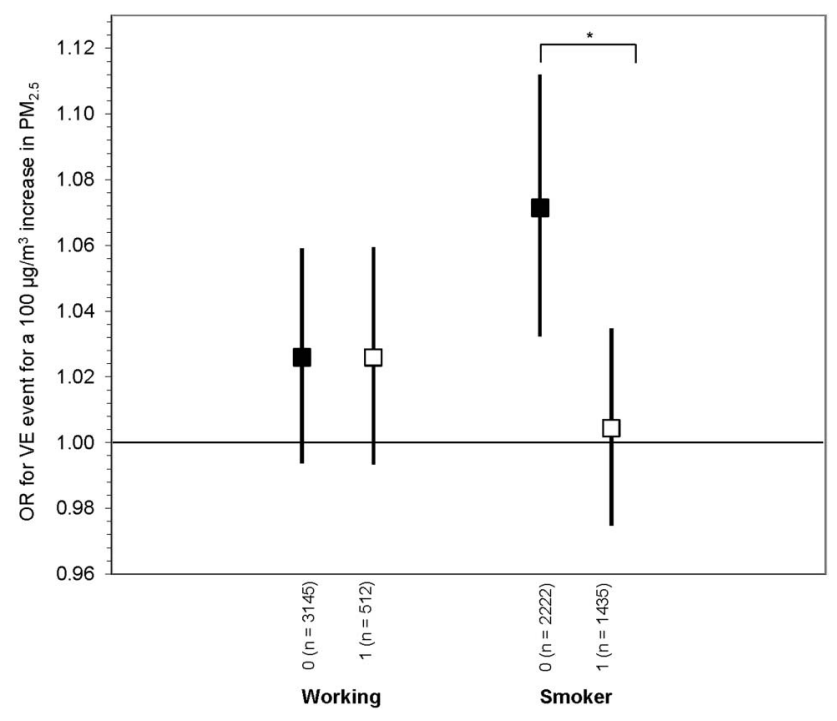

Figure 2 Effect modifiers of the association between ventricular event and $\mathrm{PM}_{2.5}$ in $6 \mathrm{~h}$ prior. $0=$ Monitoring hours with characteristic, $1=$ monitoring hours without characteristic. All models are adjusted for age, time of day, season and worksite. OR are for a $100 \mu \mathrm{g} / \mathrm{m}^{3}$ increase in $\mathrm{PM}_{2.5}$ in $6 \mathrm{~h}$ prior. Error bars indicate $95 \% \mathrm{Cls}$. ${ }^{*}$ Difference in effect is significant at $\mathrm{p}<0.05$. $\mathrm{PM}_{2.5}$, particulate matter $\leq 2.5 \mu \mathrm{m}$ aerodynamic diameter; $\mathrm{VE}$, ventricular ectopy.

shown. ${ }^{7}{ }^{28-30}$ However, not all ICD studies have found such associations. $^{8} \quad 10$ 12-14 31 Heterogeneity in the associations between PM exposures and arrhythmia response may be a reflection of the mechanisms and time course of response in different populations, as well as differences in PM sources and other co-pollutants.

Evidence supports the existence of three general pathways by which $\mathrm{PM}_{2.5}$ may affect the cardiovascular system, including the instigation of a systemic proinflammatory response, alteration in systemic autonomic nervous system balance/activity or through transmission of particles directly into circulation. ${ }^{1}$ The triggering of acute cardiovascular events may occur through multiple mechanisms including alterations in autonomic function and myocardial repolarisation, local and systemic inflammation, reactive oxygen species, coagulation and myocardial ischaemia. ${ }^{1} 2{ }^{4}$ Changes in autonomic tone may occur within a few hours, while the inflammatory and oxidative stress responses may act along a longer time scale (within several hours to days). ${ }^{1} \quad 2$ In the current study, we observed an association between PM and ectopy 6-7 h postexposure. Within the APACR study of middle-aged non-smokers, a more immediate association was observed between premature ventricular contraction (PVC) and the same and previous $30 \mathrm{~min} \mathrm{PM}_{2.5}$ exposure. ${ }^{5}$ However, at the 10th and last lag of observation, corresponding to the 5th hour postexposure, a higher (but not statistically significant) odds of $\mathrm{PM}_{2.5}$-related PVC was observed. Current study results are consistent with a study of patrol troopers exposed to traffic-related air pollution inside their patrol cars, which also demonstrated increased odds of ectopy at approximately $6-7 \mathrm{~h}$ postexposure. ${ }^{15}$

Other studies of air pollution exposures in ICD patients suggest that longer term air pollution exposures (over several days) are associated with ICD recorded events. ${ }^{31}$ In a casecrossover study of ICD patients in Boston, Massachusetts, statistically significant associations were observed between ventricular arrhythmias and moving average $\mathrm{PM}_{2.5}$ in the prior $24 \mathrm{~h}$, while increased risk, yet not statistically significant associations, were observed with $\mathrm{PM}_{2.5}$ exposures in prior 3 or $7 \mathrm{~h}^{9}$ In a study of patients with coronary heart disease, ventricular ectopy recorded by $24 \mathrm{~h}$ ECG was associated with $\mathrm{PM}_{2.5}$ exposures in the prior 48-71 h, yet not with exposures in the concurrent or prior day. ${ }^{28}$ No associations between air pollution exposures and ICD activity was observed in a study of 705 patients in London, England, ${ }^{14}$ two populations of ICD patients from Vancouver, Canada, ${ }^{8} 12$ or ICD patients from Atlanta. ${ }^{13}$ Recently, associations between arrhythmia episodes and traffic-related PM averaged over prior 3 days were observed in elderly patients within the Normative Aging Study. ${ }^{6}$

The reason for the mixed results among studies of ICD patients and air pollution exposures remains unclear, but may result from variations and limitations in study design and statistical power, or may be a reflection of effect modification with heterogeneous ICD patient populations at different risk for events. Differences in exposure, both in measurement and possible misclassification, as well as heterogeneity of exposure resulting in different toxicity may also play a role.

While PM mass concentration is associated with cardiovascular events, particle source and specific particulate constituents and characteristics, including size, appear to be important factors differentiating cardiovascular response. ${ }^{1}$ While risk of VE associated with occupational PM is greater than that of environmental PM due to higher levels of exposures in occupational settings, the effect estimates of similar magnitude found during work and non-work periods in this study suggests a loglinear relationship even at lower concentrations. Surprisingly, our results indicate that the arrhythmia response to workday exposures that are primarily dominated by welding fume exposures of small, metal-rich particles with high oxidising potential, have the same potential for an arrhythmia response as particles from an environmental source. Since this study characterised PM exposures by mass concentration alone, we could not discern the sources of PM exposures. Workday exposures may have included welding fume as well as primary or secondhand smoke. During non-work periods, exposure source may have included primary or secondhand smoke, indoor exposures from heating or cooking, and traffic or other air pollution exposures. Studies suggest a link between traffic-related exposures and arrhythmia events, ${ }^{6711}$ which may explain the comparability in potential for arrhythmia response between work and non-work PM exposures, given that participants travelled by car to the study location during the non-work study period. However, given that we lacked detailed information on the source of $\mathrm{PM}_{2.5}$, additional work is needed to support the role of particle composition in eliciting an arrhythmia response.

In this study, we observed effect modification by current smoker with a larger effect estimate for the exposure-response relationship observed among non-smokers as compared with smokers. This is contrary to the Women's Health Initiative study showing a particle-related increased odds of VE among smokers, yet no association among non-smokers. ${ }^{32}$ In the APACR study which was restricted to non-smokers, $\mathrm{PM}_{2.5}$-related VEs were observed. ${ }^{5}$ Cigarette smoking remains a leading modifiable risk factor for coronary artery disease (CAD) and sudden cardiac death (SCD) in the USA. ${ }^{33}$ Yet, the exact mechanism by which tobacco increases the risk of CAD and SCD remains unclear and studies of the arrhythmogenic effects of short-term cigarette smoking are mixed. In a crossover study of 52 smokers with ischaemic heart disease, as compared with a control period, smoking six cigarettes produced no increases in occurrence of VE. ${ }^{34}$ Likewise, in an intervention trial of 73 
patients with $\mathrm{CAD}$, in comparison to a control period of $2 \mathrm{~h}$ abstinence from smoking, during $1 \mathrm{~h}$ of smoking, no increase in VE were observed. ${ }^{33}$

The increased odds of VE due to $\mathrm{PM}_{2.5}$ exposure within this cohort were small even when expressed per $100 \mu \mathrm{g} / \mathrm{m}^{3}$ increase in $\mathrm{PM}_{2.5}$, which is quite large when compared with environmental $\mathrm{PM}_{2.5}$ concentrations which tend to be small (eg, the IQR during non-work in this population was $60 \mu \mathrm{g} / \mathrm{m}^{3}$ ). Expressed in terms of exposures observed in this cohort, the OR for a VE in an hour per IQR increase in $\mathrm{PM}_{2.5}$ in the prior $6 \mathrm{~h}$ is 1.05 (95\% CI 1.01 to 1.23$)$. It is difficult to compare the effect sizes observed in this study to other studies that reported associations between PM exposure and ectopy in healthy individuals ${ }^{15} 26$ due to differences in statistical analysis and exposure measurements. Using mixed-effects regression models evaluating the change in quantity of ectopy, a panel study of nine patrol troopers found that a $10 \mu \mathrm{g} / \mathrm{m}^{3}$ in vehicle $\mathrm{PM}_{2.5}$ exposure over a work shift, also measured using light-scattering technology, was associated with a $19 \%$ and $23 \%$ increase in quantity of hourly VE and SVE in the morning following exposure, respectively. A large cohort study evaluating ectopy over a $10 \mathrm{~s}$ period in relation to $\mathrm{PM}_{2.5}$ exposure over days among postmenopausal women found a considerably greater odds of VE over a $10 \mathrm{~s}$ period was associated with $10 \mu \mathrm{g} / \mathrm{m}^{3}$ increase in prior day $\mathrm{PM}_{2.5}$ among smokers (OR 2.00, 95\% CI 1.32 to 3.30$).^{26}$

This study is strengthened by the repeated measures study design and personal exposure monitoring, both which may have contributed to our ability to observe small particle-related effects. While our study is strengthened by personal exposure monitoring, exposure misclassification is possible. Importantly, we did not have information on the source of PM. Likewise, we did not have detailed time activity of the participants. While, we observed no difference in the exposure-response relationship between respirator and non-respirator users, we did not have detailed information about the type of respirators used (ranging from dust masks to half-face respirators) nor the timing or duration of use. Exposure misclassification may explain why respirator use did not reduce the exposure-response relationship. Likewise, exposure misclassification may also contribute to the inability to observe an exposure-response relationship among smokers.

It is unlikely that the results are explained by confounding. Results were stable when adjusted for potential confounders including heart rate, a surrogate for physical activity and stress. While air pollution contains a mixture of exposures (particles, carbon monoxide, sulfur dioxide and nitrogen dioxide) which have been implicated in health effects, ${ }^{1}$ it is unlikely that any of these pollutants would be correlated with particle exposures during both the work and non-work day exposures and are therefore unlikely confounders of the association. Furthermore, it is unlikely that results are due to chance, as consistency in the association was observed for the 6 and $7 \mathrm{~h}$ PM lags, which is consistent with biological mechanisms of particle-elicited cardiovascular response, ${ }^{1}$ as well as the time course, yet not magnitude, of response observed in the study of patrol troopers. ${ }^{15}$ It is unclear what role the healthy worker survivor effect plays in this occupational cohort where smoking or employment status can be affected by cardiovascular response to occupational exposures. Although workers who are susceptible to the cardiovascular effects of welding or smoking may leave the workforce, this would not affect the internal validity of this repeatedmeasures panel study. However, this may account for the small particle-related increased risk of ectopy observed among this healthy working population.

\section{CONCLUSIONS}

In a panel of welders with occupational and environmental exposures, we observed an association between short-term $\mathrm{PM}_{2.5}$ exposure and ventricular ectopy $6-7 \mathrm{~h}$ postexposure. Although the increased risk of exposure per unit increase in $\mathrm{PM}_{2.5}$ was small, this study suggests that individuals with no known underlying cardiovascular disease are susceptible to the arrhythmogenic effects of particle exposures.

Acknowledgements Research support by NIEHS ES009860 and ES00002. Training support by T42 OH008416, T32 ES 07069 and the Flight Attendants Medical Research Institute. The authors thank B Coull, JY Kim, S Magari, A Mehta, M McClean, E Rodrigues, R Herrick, R Hauser and the Boilermakers, Local 29, Quincy, Massachusetts, USA.

Contributors JMC contributed to all aspects of study design, data acquisition; performed data analysis; data interpretation and manuscript preparation. SCF contributed to study design, data acquisition and manuscript preparation. EAE contributed to data analysis approach, data interpretation and drafting of the manuscript. MAM contributed to data analysis approach, data interpretation and drafting of the manuscript. DCC contributed to study design, data interpretation and drafting of the manuscript.

Funding Flight Attendant Medical Research Institute; National Institute of Environmental Health Sciences; National Institute for Occupational Safety and Health.

Competing interests None declared.

Patient consent Obtained.

Ethics approval Harvard School of Public Health Institutional Review Board.

Provenance and peer review Not commissioned; externally peer reviewed.

\section{REFERENCES}

1 Brook RD, Rajagopalan S, Pope CA III, et al. American Heart Association Council on Epidemiology and Prevention, Council on the Kidney in Cardiovascular Disease, and Council on Nutrition, Physical Activity and Metabolism. Particulate matter air pollution and cardiovascular disease: an update to the scientific statement from the American Heart Association. Circulation 2010;121:2331-78.

2 Brook RD, Franklin B, Cascio W, et al. Air pollution and cardiovascular disease: a statement for healthcare professionals from the expert panel on population and prevention science of the American Heart Association. Circulation 2004; 109:2655-71.

3 Huikuri HV, Castellanos A, Myerburg RJ. Sudden death due to cardiac arrhythmias. N Engl J Med 2001;345:1473-82.

4 Link MS, Dockery DW. Air pollution and the triggering of cardiac arrhythmias. Curr Opin Cardiol 2010;25:16-22.

5 He F, Shaffer ML, Rodriguez-Colon S, et al. Acute effects of fine particulate air pollution on cardiac arrhythmia: the APACR study. Environ Health Perspect 2011;119:927-32.

6 Zanobetti A, Coull BA, Gryparis A, et al. Associations between arrhythmia episodes and temporally and spatially resolved black carbon and particulate matter in elderly patients. Occup Environ Med 2014;71:201-7.

7 Peters A, Liu E, Verrier RL, et al. Air pollution and incidence of cardiac arrhythmia. Epidemiology 2000;11:11-17.

8 Rich KE, Petkau J, Vedal S, et al. A case-crossover analysis of particulate air pollution and cardiac arrhythmia in patients with implantable cardioverter defibrillators. Inhal Toxicol 2004;16:363-72.

9 Rich DQ, Schwartz J, Mittleman MA, et al. Association of short-term ambient air pollution concentrations and ventricular arrhythmias. Am J Epidemiol 2005; 161:1123-32.

10 Rich DQ, Mittleman MA, Link MS, et al. Increased risk of paroxysmal atrial fibrillation episodes associated with acute increases in ambient air pollution. Environ Health Perspect 2006;114:120-3.

11 Dockery DW, Luttmann-Gibson H, Rich DQ, et al. Association of air pollution with increased incidence of ventricular tachyarrhythmias recorded by implanted cardioverter defibrillators. Environ Health Perspect 2005;113:670-4.

12 Vedal S, Rich K, Brauer M, et al. Air pollution and cardiac arrhythmias in patients with implantable cardioverter defibrillators. Inhal Toxicol 2004;16:353-62.

13 Metzger KB, Klein M, Flanders WD, et al. Ambient air pollution and cardiac arrhythmias in patients with implantable defibrillators. Epidemiology 2007; 18:585-92

14 Anderson HR, Armstrong B, Hajat $S$, et al. Air pollution and activation of implantable cardioverter defibrillators in London. Epidemiology 2010;21:405-13 
15 Riediker M, Cascio WE, Griggs TR, et al. Particulate matter exposure in cars is associated with cardiovascular effects in healthy young men. Am J Respir Crit Care Med 2004;169:934-40.

16 Liao D, Duan Y, Whitsel EA, et al. Association of higher levels of ambient criteria pollutants with impaired cardiac autonomic control: a population-based study. Am J Epidemio/ 2004;159:768-77.

17 Cavallari JM, Eisen EA, Chen JC, et al. Night heart rate variability and particulate exposures among boilermaker construction workers. Environ Health Perspect 2007; 115:1046-51

18 Chen JC, Cavallari JM, Stone PH, et al. Obesity is a modifier of autonomic cardiac responses to fine metal particulates. Environ Health Perspect 2007;115:1002-6.

19 Chen JC, Stone PH, Verrier RL, et al. Personal coronary risk profiles modify autonomic nervous system responses to air pollution. J Occup Environ Med 2006;48:1133-42.

20 Magari SR, Schwartz J, Williams PL, et al. The association of particulate air metal concentrations with heart rate variability. Environ Health Perspect 2002;110:875-80.

21 Magari SR, Hauser R, Schwartz J, et al. Association of heart rate variability with occupational and environmental exposure to particulate air pollution. Circulation 2001;104:986-91.

22 Ferris BG. Epidemiology Standardization Project (American Thoracic Society). Am Rev Respir Dis 1978;118(6 Pt 2):1-120.

23 Kim JY, Magari SR, Herrick RF, et al. Comparison of fine particle measurements from a direct-reading instrument and a gravimetric sampling method. I Occup Environ Hyg 2004;1:707-15.

24 Schwartz J. The distributed lag between air pollution and daily deaths. Epidemiology 2000;11:320-6.
25 Meng QY, Turpin BJ, Korn L, et al. Influence of ambient (outdoor) sources on residential indoor and personal PM2.5 concentrations: analyses of RIOPA data. J Expo Anal Environ Epidemiol 2005;15:17-28.

26 Liao D, Whitsel EA, Duan Y, et al. Ambient particulate air pollution and ectopy - the environmental epidemiology of arrhythmogenesis in Women's Health Initiative Study, 1999-2004. J Toxicol Environ Health Part A 2009;72:30-8.

27 Engel G, Cho S, Ghayoumi A, et al. Prognostic significance of PVCs and resting heart rate. Ann Noninvasive Electrocardiol 2007;12:121-9.

28 Berger A, Zareba W, Schneider A, et al. Runs of ventricular and supraventricular tachycardia triggered by air pollution in patients with coronary heart disease. J Occup Environ Med 2006;48:1149-58.

29 Sarnat SE, Suh HH, Coull BA, et al. Ambient particulate air pollution and cardiac arrhythmia in a panel of older adults in Steubenville, Ohio. Occup Environ Med 2006;63:700-6

30 Ljungman PL, Berglind N, Holmgren C, et al. Rapid effects of air pollution on ventricular arrhythmias. Eur Heart J 2008;29:2894-901.

31 Rich DQ, Kim MH, Turner JR, et al. Association of ventricular arrhythmias detected by implantable cardioverter defibrillator and ambient air pollutants in the St Louis, Missouri metropolitan area. Occup Environ Med 2006;63:591-6.

32 Centers for Disease Control and Prevention (CDC). Annual smoking-attributable mortality, years of potential life lost, and economic costs-United States, 1995-1999. MMWR Morb Mortal Wkly Rep 2002;51:300-3.

33 Myers MG, Benowitz NL, Dubbin JD, et al. Cardiovascular effects of smoking in patients with ischemic heart disease. Chest 1988;93:14-19.

34 Davis MJ, Hockings BE, el Dessouky MA, et al. Cigarette smoking and ventricular arrhythmia in coronary heart disease. Am J Cardiol 1984;54:282-5. 